\title{
Effects of essential amino acids on lipid metabolism in mice and humans
}

\author{
Fei Xiao', Ying Du', Ziquan Lv', Shanghai Chen', Jianmin Zhu², Hongguang Sheng² \\ and Feifan Guo'
}

${ }^{1}$ Key Laboratory of Nutrition and Metabolism, Institute for Nutritional Sciences, Shanghai Institute for Biological Sciences, Chinese Academy of Sciences, The Graduate School of the Chinese Academy of Sciences, Shanghai, China 2Shanghai Xuhui Central Hospital, Shanghai, China
Correspondence should be addressed to F Guo

Email

ffguo@sibs.ac.cn

\begin{abstract}
Eight amino acids are considered essential for human nutrition, and three of them, including leucine, isoleucine and valine, are called as branched-chain amino acids (BCAAs). We recently discovered that dietary deficiency of any BCAA for 7 days rapidly reduces the abdominal fat mass in mice. The goal of this study was to investigate (1) whether dietary deficiency of the other five essential amino acids (EAAs), including phenylalanine, threonine, tryptophan, methionine and lysine, would produce similar effects and (2) whether an association between serum AAs and obesity was observed in humans in Chinese Han population. Similar to BCAAs deprivation, dietary deficiency of any of these five EAAs for 7 days significantly reduced abdominal fat mass, which is likely caused by increased energy expenditure. Expression of genes and proteins related to lipolysis, however, were differentially regulated by different EAAs. These results suggest a crucial role of EAAs deprivation on lipid metabolism in mice. Our human studies revealed that levels of four EAAs (leucine, isoleucine, valine and phenylalanine) were elevated in obese humans compared with those in lean controls in Chinese Han population. Based on the results obtained from mice, we speculate that these four EAAs might play important roles in human obesity.
\end{abstract}

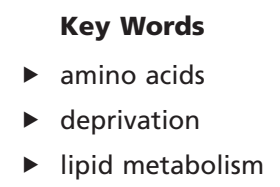

Journal of Molecular

Endocrinology

(2016) 57, 223-231

\section{Introduction}

Obesity has become a leading public health problem in many countries around the world. One of the important factors that contributes to obesity is dietary macronutrient including fat, glucose and amino acids (AAs) (Attie \& Scherer 2009). The effects of fat and glucose on lipid metabolism regulation have been well known; however, the effects of AAs are poorly understood. AAs are the building blocks of proteins and have also been shown to regulate many cell signaling pathways (Nair \& Short 2005). Eight amino acids, including phenylalanine (Phe), valine (Val), threonine (Thr), tryptophan (Trp), isoleucine (Ile), methionine (Met), leucine (Leu) and lysine (Lys), are considered as essential amino acids (EAAs) for human nutrition, as they could not be synthesized by the body and should be obtained from diet or degradation from protein. Among all EAAs, Leu, Ile and Val are called as branched-chain amino acids (BCAAs), and all of them have non-linear aliphatic side chains. As EAA plays a major role in various physiological processes, such as reproduction, recovery and brain function, altered levels of any specific EAA might result in significant changes in whole body metabolism.

Increasing evidence has shown that BCAAs play important roles in lipid metabolism regulation

Published by Bioscientifica Ltd 
(Zhang et al. 2007, Nairizi et al. 2009, Nishimura et al. 2010). By feeding mice a diet deficient of any BCAA, we recently demonstrated that BCAA deprivation has a significant impact on decreasing abdominal fat mass and improving insulin sensitivity (Guo \& Cavener 2007, Cheng et al. 2010, Xiao et al. 2011, Du et al. 2012). In addition, some of these effects are regulated by a pathway dependent on an AA sensor named general control non-depressible (GCN)2 (Guo \& Cavener 2007, Xiao et al. 2011), which is activated in response to any EAA deficiency (Zhang et al. 2002). The effects of deprivation of the other five EAAs on lipid metabolism, however, remain to be explored. Furthermore, recent studies have indicated a different BCAA-related AA metabolic signature between obese and lean subjects in Western population (Newgard et al. 2009). As many aspects, including lifestyle and food resources contributing to AA metabolism and obesity, are different between Chinese and Western population, whether this AA metabolic signature exists in Chinese Han population is unknown. For these reasons, the aim of this study was to (1) investigate whether deficiency of the other five EAAs, including Phe, Thr, Trp, Met and Lys, would produce similar effects and (2) whether an association between serum AAs and obesity was observed in humans in Chinese Han population.

As observed for BCAAs deprivation, dietary deficiency of the other five EAAs significantly reduced abdominal fat mass, which was also likely caused by increased energy expenditure. In contrast, lipolysis-related genes and proteins were differentially regulated by different EAAs. These results suggest a crucial role of EAAs in lipid metabolism regulation in mice. Consistent with these results, our human studies revealed that levels of four essential amino acids (Leu, Ile, Val and Phe) and eight non-essential amino acids were significantly different in obese humans compared with those in lean controls in Chinese Han population. Based on the results obtained from mice, we speculate that these four EAAs might play important roles in human obesity.

\section{Materials and methods}

\section{Subjects}

The population of this study consisted of 100 unrelated Shanghai residents (77 men and 23 women) from Shanghai Xuhui Central Hospital, Shanghai, China. All participants attended a physical examination during which standard anthropometric measurements and fasting blood samples were collected. Those with severe psychological disorders; physical disabilities; cancer; cardiovascular disease; Alzheimer's disease or currently diagnosed with tuberculosis, AIDS and other communicable diseases were excluded. All participants were recruited after written consent was approved by Shanghai Xuhui Central Hospital. Body weight and height were measured, and body mass index (BMI) was calculated as weight $(\mathrm{kg}) /$ height $\left(\mathrm{m}^{2}\right)$. Overweight/obesity and normal weight were defined as $\mathrm{BMI} \geq 24.0 \mathrm{~kg} / \mathrm{m}^{2}$ and $18.0 \leq \mathrm{BMI}<24.0 \mathrm{~kg} / \mathrm{m}^{2}$, respectively, which was modified from recommendation by the Working Group on Obesity in China (Wu 2006).

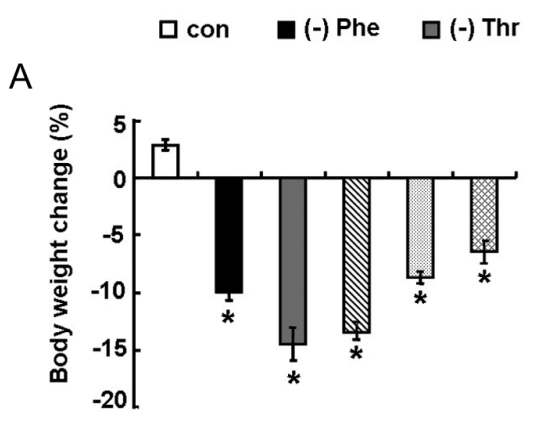

C

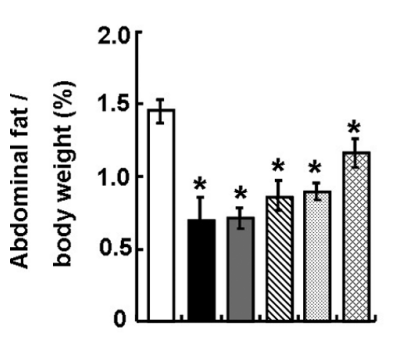

D

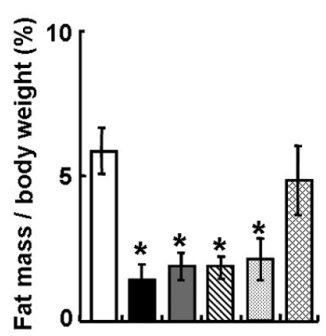

$B$

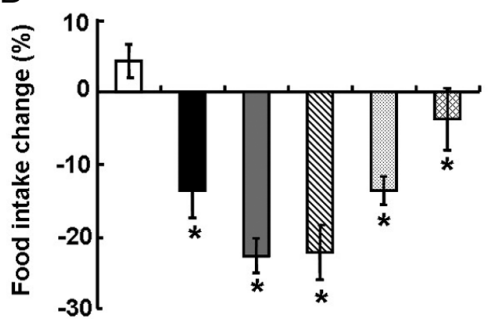

E

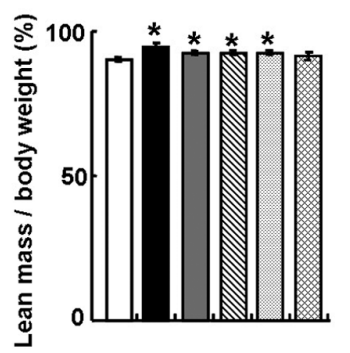

\section{Figure 1}

The effects on body weight, fat weight and food intake by deficiency of Phe, Thr, Trp, Met and Lys. Mice were fed a control diet or diet deficient of Phe, Thr, Trp, Met or Lys for 7 days. Data are mean \pm S.E.M. of at least two independent experiments with mice of each diet for each experiment ( $n=5-6$ for each group). Statistical significance was determined by the two-tail Student's $t$-test for the effect of any deficient diet vs control diet $\left({ }^{*} P<0.05\right)$. (A) Body weight reduction; (B) food intake change; (C) abdominal fat in proportion to body weight and (D and E) body composition measured with NMR. http://jme.endocrinology-journals.org DOI: 10.1530/JME-16-0116
๑) 2016 Society for Endocrinology Printed in Great Britain
Published by Bioscientifica Ltd 
Table 1 Serum measurements in mice maintained on different diets. ${ }^{*} P<0.05$.

\begin{tabular}{|c|c|c|c|c|c|c|}
\hline & Control & (-) Phe & (-) Thr & (-) Trp & (-) Met & (-) Lys \\
\hline Norepinephrine (ng/L) & $140.31 \pm 21.81$ & $260.07 \pm 14.52 *$ & $187.62 \pm 14.48 *$ & $296.05 \pm 14.02 *$ & $190.07 \pm 10.50 *$ & $272.41 \pm 14.51$ * \\
\hline TG (mmol/L) & $0.14 \pm 0.15$ & $0.08 \pm 0.02 *$ & $0.10 \pm 0.01 *$ & $0.08 \pm 0.01 *$ & $0.09 \pm 0.01 *$ & $0.09 \pm 0.02 *$ \\
\hline Cholesterol (mmol/L) & $2.66 \pm 0.10$ & $2.12 \pm 0.06 *$ & $2.23 \pm 0.06 *$ & $2.05 \pm 0.06 *$ & $1.70 \pm 0.09 *$ & $\begin{array}{r}2.33 \pm 0.03 \\
(P=0.069)\end{array}$ \\
\hline
\end{tabular}

\section{Animals and diets}

Wild-type male C57BL/6J mice were obtained from Shanghai Laboratory Animal Co, Ltd. (SLAC, Shanghai, China). Eight- to ten-week-old mice were housed in laboratory cages under a $12 \mathrm{~h}$ light: $12 \mathrm{~h}$ darkness cycle at $25^{\circ} \mathrm{C}$ and were maintained on a regular commercial rodent chow diet before experiments. Control (nutritionally complete amino acid), (-) Phe (phenylalanine deficient), (-) Thr (threonine deficient), (-) Trp (tryptophan deficient), (-) Met (methionine deficient) and (-) Lys (lysine deficient) diets were obtained from Research Diets, Inc (New Brunswick, NJ, USA). All diets were isocaloric and compositionally the same in terms of carbohydrate and lipid component. Animal studies were conducted with experimental procedures as described previously (Guo \& Cavener 2007, Cheng et al. 2010, Du et al. 2012). Mice were fed a control diet or diet deficient for Phe, Thr, Trp, Met or Lys for 7 days. All the animals were killed at the same time of light cycle-1400h. All animal experiments were performed using protocols approved by the Institutional Animal Care and Use Committee of the Institute for Nutritional Sciences, Sibs, CAS and in conformity with the Public Health Service Policy on Humane Care and Use of Laboratory Animals.

\section{Indirect calorimetry}

After 6 days of feeding with control, (-) Phe, (-) Thr, (-) Trp, (-) Met or (-) Lys diet, oxygen consumption and physical activity of mice were determined by a comprehensive lab animal monitoring system (Columbus Instruments, Columbus, OH, USA) for $24 \mathrm{~h}$ according to the manufacturer's instructions (Cheng et al. 2010, Du et al. 2012).

\section{Serum measurements}

Serum was obtained as described previously (Guo \& Cavener 2007, Cheng et al. 2010, Du et al. 2012).

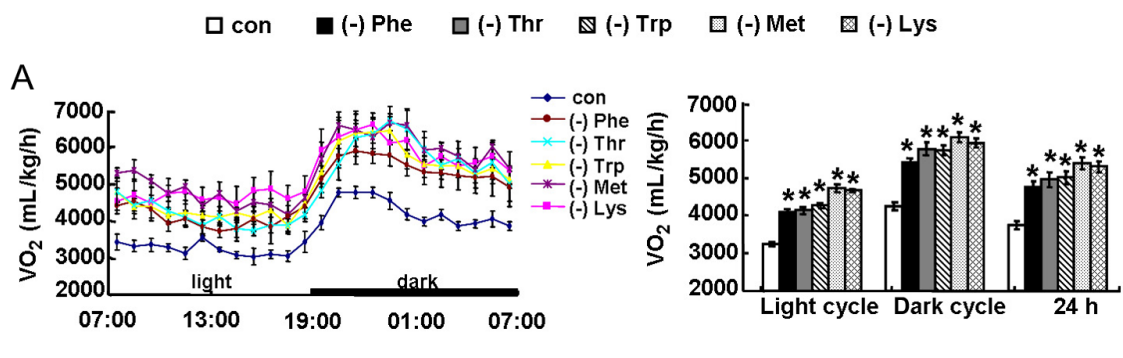

B
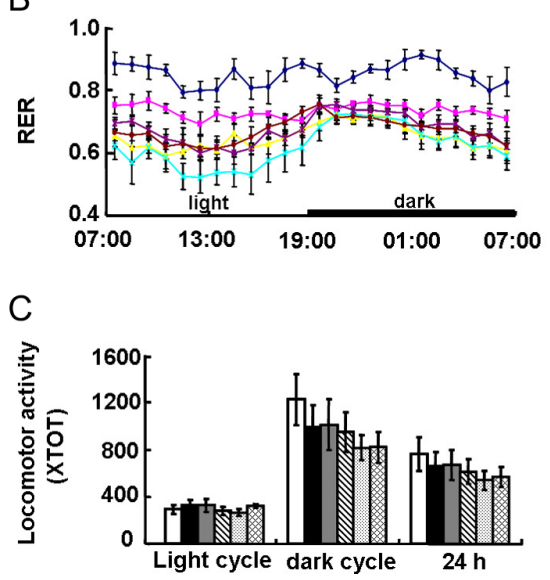
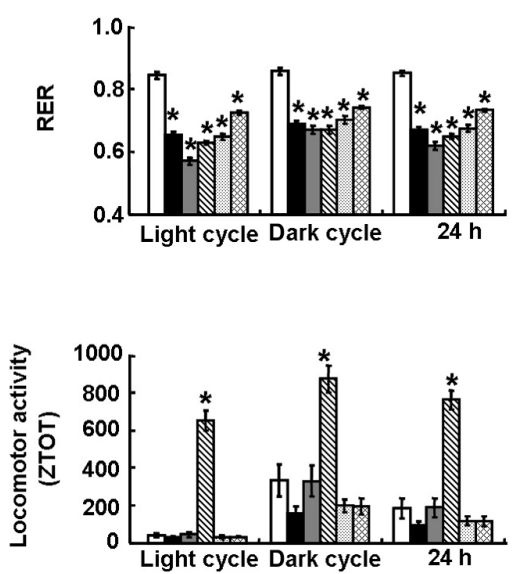

Figure 2

The effects on energy expenditure by deficiency of Phe, Thr, Trp, Met and Lys. The energy expenditure was measured by indirect calorimetry in mice fed a control diet or diet deficient of Phe, Thr, Trp, Met or Lys for 7 days over 24-48 h after 6-h acclimation to the metabolic chamber. Data are mean \pm S.E.M. of at least two independent experiments with mice of each diet for each experiment ( $n=5-6$ for each group). Statistical significance was determined by the two-tail Student's $t$-test for the effect of any deficient diet vs control diet $\left({ }^{*} P<0.05\right)$. (A) 24-h oxygen consumption; (B) respiratory exchange ratio (RER) and (C) physical activity. A full colour version of this figure is available at http://dx.doi.org/ 10.1530/JME-16-0116. http://jme.endocrinology-journals.org DOI: 10.1530/JME-16-0116
() 2016 Society for Endocrinology Printed in Great Britain
Published by Bioscientifica Ltd 


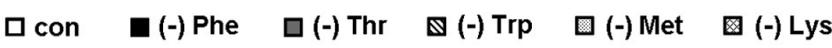
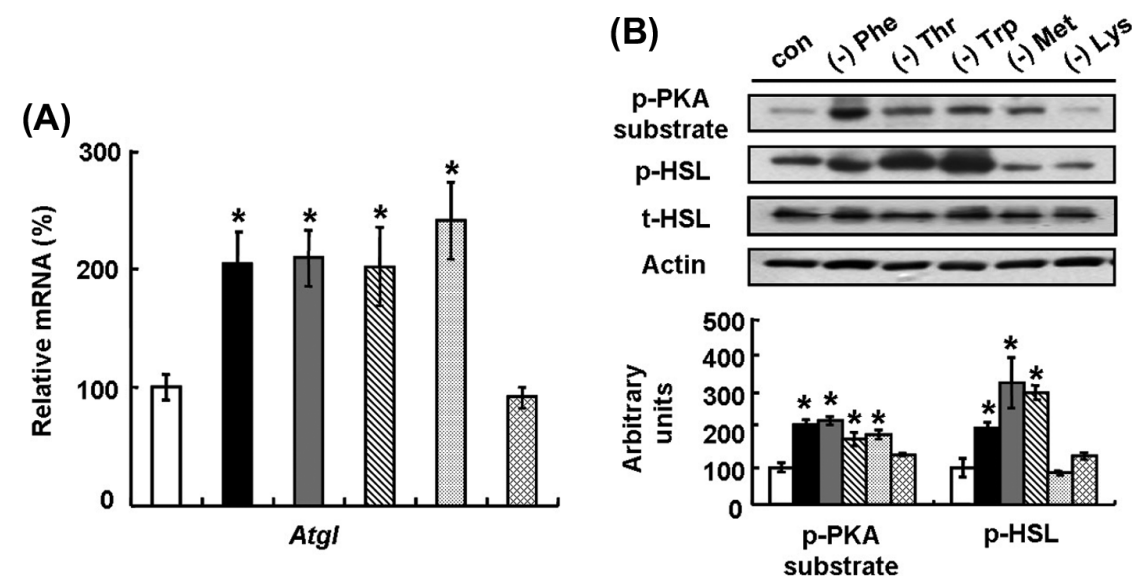

Figure 3
The effects on genes and proteins regulating
lipolysis in WAT by deficiency of Phe, Thr, Trp, Met
and Lys. Mice were fed a control diet or diet
deficient of Phe, Thr, Trp, Met or Lys for 7 days.
Data are mean \pm S.E.M. of at least two independent
real-time PCR experiments (A) or western blot (B)
with mice of each diet for each experiment
( $n=5-6$ for each). Statistical significance was
determined by the two-tail Student's $t$-test for
the effect of any deficient diet vs control diet
( $P<0.05)$. (A) Atgl mRNA; (B) p-PKA substrate
proteins and p-HSL (top western blot; bottom,
p-HSL and p-PKA substrate proteins relative
to actin).

Amino acid (AA) levels were determined by standard ion exchange chromatography using a Beckman 6300 automated amino acid analyzer. Serum triglyceride (TG), cholesterol and norepinephrine (NE) were determined using triglyceride kit (BTKH Clinical Reagents, Beijing, China), cholesterol kit (BTKH Clinical Reagents) and enzyme-linked immunosorbent assay (ELISA) kit (R\&D Systems), respectively. These assays were conducted according to manufacturer's instructions.

\section{Western blot analysis}

Western blot was performed as described previously (Cheng et al. 2010). Primary antibodies anti-p-HSL and anti-p-PKA substrate antibodies were purchased from Cell Signaling; anti-actin antibody was from Sigma. Band intensities were measured using Quantity One (Bio-Rad Laboratories) and normalized to actin.

\section{RNA isolation and relative quantitative RT-PCR}

Total RNA from mouse tissues was isolated as described previously (Cheng et al. 2010, Du et al. 2012). Realtime quantitative PCR was performed with SYBR Green I Master Mix reagent by ABI 7500 system (Applied Biosystems), using GAPDH as an internal control for normalization. The primers for Atgl were as follows: sense 5'-GTGAAGCAGGTGCCAACATTATTG-3', antisense 5'-AAACACGAGTCAGGGAGATGCC-3'.

\section{Statistics}

All data are expressed as mean \pm S.E.m. Significant differences between (-) Phe, (-) Thr, (-) Trp, (-) Met or (-) Lys and control group were assessed using the two-tailed
Student's t-test. For the human profiling data, statistical analysis was carried out using STATA. The level of amino acid in obese and lean subjects was compared using multi-factor variance analysis (ANOVA) with or without adjustment for age and sex. $P<0.05$ was considered statistically significant.

\section{Results}

The effects on body weight and fat mass by deficiency of Phe, Thr, Trp, Met or Lys

We previously have shown that mice maintained on a BCAA-deficient diet undergo rapid loss of abdominal fat (Cheng et al. 2010, Du et al. 2012). The goal of this study was to investigate whether the other five EAAs deficiency produce similar effects in mice. For this purpose, mice were fed a control diet or diet deficient for Phe, Thr, Trp, Met or Lys for 7 days, a duration that is comparable with those during BCAAs deprivation. Deficiency of any of these five EAAs for 7 days resulted in significant reduction in body weight, to the smallest extent by Lys deprivation, compared with mice maintained on a control diet (Fig. 1A). Similar changes were observed for abdominal fat mass (Fig. 1C). Consistent with these results, total body fat was decreased significantly by the deprivation of Phe, Thr, Trp or Met in comparison with

Table 2 Baseline characteristics of the study population.

\begin{tabular}{|c|c|c|}
\hline & Lean & Obese \\
\hline Baseline characteristic & $n=20$ & $n=80$ \\
\hline Age (years) & $32.7 \pm 1.63$ & $33.1 \pm 0.68$ \\
\hline Male & $4(20 \%)$ & $73(91.25 \%)$ \\
\hline Female & $16(80 \%)$ & $7(8.75 \%)$ \\
\hline BMI & $18.9 \pm 0.27$ & $29.6 \pm 0.23$ \\
\hline
\end{tabular}

Published by Bioscientifica Ltd 
the control mice as measured by NMR (Fig. 1D). Although not statistically significant, total fat mass also showed decreased tendency in Lys-deprived mice compared with control mice (Fig. 1D). There was a small increase in the proportion of lean mass in Phe, Thr, Trp or Met-deprived mice compared with that in the control group (Fig. 1E). Deficiency of Phe, Thr, Trp and Met also significantly reduced the brown fat mass. Brown fat mass remained the same in Lys-deprived mice.

Similar to those observed during BCAA deprivation, we found that serum levels of triglyceride (TG) and cholesterol were decreased, and serum norepinephrine (NE) levels were increased, in mice maintained on these five amino acids-deficient diet compared with control groups (Table 1).

\section{The effects on food intake and energy expenditure by deficiency of Phe, Thr, Trp, Met or Lys}

As decreased fat mass is caused by reduced food intake and/or increased energy expenditure, we compared the food intake among different groups. Deprivation of these 5 essential amino acids significantly decreased food intake, with the smallest extent by Lys deprivation, compared with mice maintained on the control diet (Fig. 1B).

Table 3 Amino acids profiles.

\begin{tabular}{|c|c|}
\hline & Lean \\
\hline \multicolumn{2}{|l|}{ Amino acids ( $\mu \mathrm{mol} / \mathrm{L})$} \\
\hline Phosphoserine (pSer) & $0.54 \pm 0.05$ \\
\hline Phosphoethanolamine (pEtn) & $2.11 \pm 0.47$ \\
\hline Taurine (Tau) & $145.39 \pm 12.09$ \\
\hline Asparagine (Asn) & $90.31 \pm 4.35$ \\
\hline Serine (Ser) & $215.54 \pm 6.04$ \\
\hline Hydroxyproline (Hyp) & $18.39 \pm 1.66$ \\
\hline Glycine (Gly) & $384.18 \pm 18.33$ \\
\hline Glutamine (Gln) & $830.08 \pm 37.07$ \\
\hline Aspartic Acid (Asp) & $23.38 \pm 1.21$ \\
\hline Ethanolamine (Etn) & $176.35 \pm 1.79$ \\
\hline Histidine (His) & $102.46 \pm 2.97$ \\
\hline Threonine (Thr) & $181.54 \pm 8.88$ \\
\hline Citrulline (Cit) & $35.98 \pm 1.56$ \\
\hline Sarcosine (Sar) & $2.28 \pm 0.13$ \\
\hline$\beta$-Alanine (bAla) & $16.93 \pm 2.05$ \\
\hline Alanine (Ala) & $527.63 \pm 28.01$ \\
\hline Glutamate (Glu) & $61.63 \pm 4.21$ \\
\hline 1-Methyl-histidine (1MHis) & $10.30 \pm 1.74$ \\
\hline 3-Methyl-histidine (3MHis) & $3.81 \pm 0.26$ \\
\hline Argininosuccinate (Asa) & $248.71 \pm 13.04$ \\
\hline Carnosine (Car) & $0.45 \pm 0.09$ \\
\hline Anserine (Ans) & $0.71 \pm 0.10$ \\
\hline Homocitrulline (Hcit) & $0.61 \pm 0.09$ \\
\hline Arginine (Arg) & $134.40 \pm 4.34$ \\
\hline$\alpha$-Aminoadipic acid (Aad) & $1.70 \pm \pm 0.08$ \\
\hline$\beta$-Aminoisobutyric acid (bAib) & $2.45 \pm 0.34$ \\
\hline$\alpha$-Aminobutyric acid (Abu) & $26.90 \pm 2.46$ \\
\hline 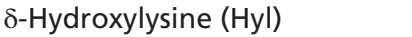 & $1.24 \pm 0.15$ \\
\hline Proline (Pro) & $191.59 \pm 16.00$ \\
\hline Ornithine (Orn) & $112.22 \pm 9.49$ \\
\hline Cystathionine (Cth) & $3.44 \pm 0.34$ \\
\hline Cystine (Cys) & $24.16 \pm 1.73$ \\
\hline Lysine (Lys) & $244.15 \pm 11.70$ \\
\hline Methionine (Met) & $42.07 \pm 1.57$ \\
\hline Valine (Val) & $280.54 \pm 11.51$ \\
\hline Tyrosine (Tyr) & $77.08 \pm 2.82$ \\
\hline Homocysteine (Hcy) & $4.68 \pm 0.37$ \\
\hline Isoleucine (Ile) & $74.78 \pm 3.22$ \\
\hline Leucine (Leu) & $148.11 \pm 4.94$ \\
\hline Phenylalanine (Phe) & $82.86 \pm 2.24$ \\
\hline Tryptophan (Trp) & $59.50 \pm 2.36$ \\
\hline
\end{tabular}

\begin{tabular}{c}
\hline Obese \\
\hline $0.61 \pm 0.03$ \\
$2.58 \pm 8.91$ \\
$136.06 \pm 8.91$ \\
$92.27 \pm 1.87$ \\
$200.73 \pm 5.69$ \\
$20.36 \pm 0.94$ \\
$328.51 \pm 5.43$ \\
$858.31 \pm 10.89$ \\
$29.10 \pm 0.98$ \\
$178.22 \pm 1.08$ \\
$111.38 \pm 1.33$ \\
$167.68 \pm 2.84$ \\
$35.60 \pm 0.91$ \\
$2.38 \pm 0.08$ \\
$22.16 \pm 0.99$ \\
$653.31 \pm 14.25$ \\
$108.04 \pm 3.31$ \\
$12.85 \pm 0.59$ \\
$4.91 \pm 0.13$ \\
$295.96 \pm 5.15$ \\
$0.44 \pm 0.04$ \\
$0.63 \pm 0.05$ \\
$0.88 \pm 0.06$ \\
$138.52 \pm 2.67$ \\
$2.79 \pm 0.07$ \\
$2.15 \pm 0.17$ \\
$28.38 \pm 0.89$ \\
$1.26 \pm 0.09$ \\
$231.47 \pm 6.54$ \\
$134.35 \pm 4.48$ \\
$2.95 \pm 0.25$ \\
$37.32 \pm 1.20$ \\
$292.55 \pm 4.71$ \\
$42.51 \pm 1.18$ \\
$366.17 \pm 6.74$ \\
$95.38 \pm 1.71$ \\
$4.23 \pm 0.19$ \\
$106.28 \pm 2.14$ \\
$207.84 \pm 3.84$ \\
$99.79 \pm 1.57$ \\
$64.57 \pm 1.04$ \\
\hline
\end{tabular}

\begin{tabular}{cccc}
\hline \multicolumn{4}{c}{ P value adjusted for } \\
\hline- & Age & Sex & Age and sex \\
0.3353 & 0.4208 & 0.8248 & 0.8584 \\
0.4639 & 0.3834 & 0.4021 & 0.3663 \\
0.6218 & 0.6073 & 0.5014 & 0.4730 \\
0.6523 & 0.7532 & 0.9168 & 0.8979 \\
0.2126 & 0.1611 & 0.1591 & 0.1379 \\
0.3395 & 0.3686 & 0.1772 & 0.1869 \\
$<0.001$ & $<0.001$ & $<0.001$ & $<0.001$ \\
0.3240 & 0.3916 & 0.1947 & 0.1616 \\
0.0066 & 0.0103 & 0.0829 & 0.0936 \\
0.4248 & 0.4530 & 0.4105 & 0.4022 \\
0.0043 & 0.0077 & 0.9887 & 0.9493 \\
0.0566 & 0.0559 & 0.1049 & 0.0903 \\
0.8499 & 0.7997 & 0.0422 & 0.0351 \\
0.5436 & 0.6007 & 0.4876 & 0.4907 \\
0.0213 & 0.0218 & 0.0216 & 0.0156 \\
$<0.001$ & $<0.001$ & 0.0232 & 0.0246 \\
$<0.001$ & $<0.001$ & $<0.001$ & $<0.001$ \\
0.0834 & 0.0824 & 0.9122 & 0.9019 \\
$<0.001$ & $<0.001$ & 0.4728 & 0.4716 \\
$<0.001$ & $<0.001$ & 0.3933 & 0.4377 \\
0.9733 & 0.8948 & 0.9982 & 0.9683 \\
0.4714 & 0.4020 & 0.2901 & 0.2908 \\
0.0357 & 0.6797 & 0.8580 & 0.8723 \\
0.4764 & 0.5199 & 0.7003 & 0.6987 \\
$<0.001$ & $<0.001$ & $<0.001$ & $<0.001$ \\
0.4342 & 0.5019 & 0.0675 & 0.0664 \\
0.4946 & 0.5705 & 0.7656 & 0.7074 \\
0.9043 & 0.9955 & 0.1811 & 0.2043 \\
0.0106 & 0.0130 & 0.1104 & 0.1143 \\
0.0315 & 0.0280 & 0.9692 & 0.9049 \\
0.3527 & 0.4314 & 0.5163 & 0.5074 \\
$<0.001$ & $<0.001$ & 0.0075 & 0.0072 \\
$<0.001$ & $<0.001$ & 0.4651 & 0.5232 \\
0.8603 & 0.9748 & 0.4948 & 0.4292 \\
$<0.001$ & $<0.001$ & 0.0189 & 0.0220 \\
$<0.001$ & $<0.001$ & $<0.001$ & 0.0011 \\
0.2891 & 0.3377 & 0.2365 & 0.2282 \\
$<0.001$ & $<0.001$ & 0.0047 & 0.0054 \\
$<0.001$ & $<0.001$ & 0.0028 & 0.0033 \\
$<0.001$ & $<0.001$ & 0.0079 & 0.0093 \\
0.0354 & 0.0391 & 0.3502 & 0.3680 \\
\hline & & & \\
\hline & & \\
0.037 &
\end{tabular}


We have previously shown that the decreased fat mass during BCAA deprivation is caused by increased energy expenditure (Cheng et al. 2010, Du et al. 2012). Therefore, we measured the energy expenditure by indirect calorimetry in mice under different diets. The total energy expenditure (24-h $\mathrm{O}_{2}$ consumption, normalized to lean body mass) was markedly increased in all these five EAA-deprived mice compared with that in mice maintained on a control diet (Fig. 2A). The respiratory exchange ratio $\left(\mathrm{RER}, \mathrm{VCO}_{2} / \mathrm{VO}_{2}\right.$ ) was also lower in these mice during both darkness and light phases (Fig. 2B). Similar to BCAA deprivation (Cheng et al. 2010, Du et al. 2012), locomotor activity was not affected by deficiency of any of these five EAAs compared with control mice (Fig. 2C). By contrast, locomotor activity in $\mathrm{z}$ axis was significantly increased in Trp-deprived mice compared with that in control mice (Fig. 2C).

\section{The effects on lipolysis in WAT by deficiency of Phe, Thr, Trp, Met or Lys}

We have previously shown that deficiency of BCAAs stimulates lipolysis in WAT (Cheng et al. 2010, Du et al. 2012). To investigate whether this is a general effect of all EAAs, we examined the changes in genes and proteins related to lipolysis in WAT of mice maintained on a control diet or diet deficient for Phe, Thr, Trp, Met or Lys. mRNA levels of adipose triglyceride lipase (Atgl), the first enzyme regulating lipolysis (Schoenborn et al. 2006), were significantly increased by Phe, Thr, Trp and Met deprivation compared with those maintained on a control diet (Fig. 3A). Deprivation of these four EAAs also increased the levels of phosphorylated hormonesensitive lipase (HSL), the key enzyme regulating lipolysis (Martin et al. 2009), in WAT compared with control mice (Fig. 3B). Consistent with increased levels of phosphorylated HSL, levels of phosphorylated substrate for PKA, the kinase that phosphorylates HSL (Yeaman 1990), were also elevated in WAT of these mice (Fig. 3B). Lys deprivation, however, has no effect on levels of Atgl mRNA and phosphorylation of HSL and PKA in WAT compared with control mice (Fig. 3A and B).

\section{Amino acids profiling in Chinese population}

Based on our animal studies that suggest a close relationship between AAs and lipid metabolism, we were interested in exploring whether this correlation exists in Chinese Han population. We therefore measured plasma levels of AAs in obese and lean subjects recruited from
Shanghai as examples for Chinese Han population. The characteristics of the 100 participants are presented in Table 2. Eighty obese and twenty lean subjects underwent baseline evaluation. The obese subjects comprised $91.25 \%$ men and $8.75 \%$ women, whereas the lean subjects were $20 \%$ men and $80 \%$ women. The average age of the obese subjects was 33.1 years, and their BMI was $29.6 \mathrm{~kg} / \mathrm{m}^{2}$, compared with 32.7 years and $18.9 \mathrm{~kg} / \mathrm{m}^{2}$ for the lean controls (Table 2). After adjustment for age and sex, among a total of 41 amino acids measured in the serum, levels of 10 amino acids were found to be dramatically elevated in obese compared with lean participants ( $\beta$-alanine (bAla), alanine (Ala), glutamate (Glu), $\alpha$-aminoadipic acid (Aad), cystine (Cys), tyrosine (Tyr), Val, Ile, Leu and Phe; $P \leq 0.05$ ), whereas citrulline (Cit) and glycine (Gly) levels were lower in obese subjects Table 3 ).

\section{Discussion}

Although human studies have implicated an association between AAs and obesity, the causal link is not fully understood. Thus, animal studies have been conducted by providing animals with increased or decreased amounts of AAs in diet or drinking water. Regarding lipid metabolism regulation, the effects of BCAAs are the most noticed so far, although results are not always the same. For example, some studies showed that increased BCAAs prevent obesity and hyperglycemia in mice (Zhang et al. 2007, Nishimura et al. 2010), whereas others reported that increased BCAAs either have no effect or lead to obesity (Nairizi et al. 2009, Newgard et al. 2009). By feeding mice with a diet deficient for individual BCAA, we found that BCAA deficiency rapidly decreases fat mass (Cheng et al. 2010, Du et al. 2012) and improves insulin sensitivity (Xiao et al. 2011). In contrast to extensive studies about the effects of BCAAs on lipid metabolism, the effects of the other five EAAs, however, remain largely unknown. Here, we showed that dietary deficiency of Phe, Thr, Trp, Met or Lys also reduces fat mass, in a similar manner as observed during BCAAs deprivation. These results suggest an important role of EAAs on lipid metabolism regulation. The extent of fat loss, however, varies under deficiency of different EAAs, which could be due to the specific character of each AA.

Consistent with our results of Met and Thr deficiency, other studies have shown that Met restriction decreases fat mass in rats (Malloy et al. 2006, Hasek et al. 2010) and Thr deficiency decreases lipid content in rat kidney (Singh 1979). A possible link between Trp and lipid metabolism has been implicated by a previous study showing that

Published by Bioscientifica Ltd. 
lipids content in liver were significantly decreased as the levels of dietary Trp increased in growing broiler chicks (Rogers \& Pesti 1990). In addition, very little has been known about the effect of Trp on lipid metabolism regulation. Therefore, this study has provided important direct evidence for an effect of Trp deficiency on lipid metabolism in adipose tissue. Furthermore, the decreased fat mass in Trp-deprived mice may also be stimulated by increased locomotor activity, which is a unique character distinguished from other EAAs deficiency. Consistent with these results, studies have shown that Trp deletion increased the total distance traveled in an animal model of schizophrenia by about 42\% (Bubenikova et al. 2004). Phe is the other essential amino acid, which exhibited increased levels, in addition to BCAA, in obese people in Western populations (Newgard et al. 2009), indicating its possible strong association with lipid metabolism. Consistent with this possibility, we observed significant reduction of fat mass in mice under a diet deficient for Phe.

The extent of fat loss, however, is the smallest by Lys deprivation compared with deprivation of the other EAAs. It is not that surprising as some studies have shown that either thermogenesis or serum lipid profile was not changed by removing or addition of Lys from the diet (Smriga et al. 2000, Rathore et al. 2010, Hlais et al. 2012). There are several factors that may contribute to the smaller effect of Lys deprivation. It is shown that lipolysis and energy expenditure are stimulated by the activation of SNS and release of NE (Lowell \& Bachman 2003). In addition, multiple hormones also play important roles in the regulation of lipolysis and energy expenditure and one of such examples is insulin (Zhang et al. 2005). In this study, we found that levels of serum NE and phosphorylated PKA substrates in white adipose tissue were increased in Phe, Thr, Trp and Met-deprived mice, suggesting increased SNS activity. Furthermore, based on our previous observation showing that serum insulin levels were reduced in leucine-deprived mice (Xiao et al. 2011), we speculated that deprivation of these EAAs may have similar effects. Therefore, the activation of SNS and decreased levels of serum insulin may both contribute to the increased lipolysis and energy expenditure in mice under deprivation of each EAA. In contrast to obviously stimulated lipoysis in WAT by deficiency of other EAAs, Lys deficiency has no effect on lipolysis as demonstrated by the unaltered levels of Atgl mRNA and HSL phosphorylation in WAT compared with control mice. Because energy expenditure and serum NE levels are increased by Lys deprivation, we speculated that SNS activity is also increased in Lys-deprived mice. The increased SNS activity, however, did not lead to stimulated lipolysis, implicating that Lys might have a direct effect on lipolysis. Another reason may come from much less inhibitory effects on food intake by Lys deprivation compared with other EAAs, which is possibly caused by the differences in signaling pathways controlling food intake and time required to sense deficiency of different amino acids. It has been well established that the suppressed food intake by amino acid deficiency is regulated by GCN2-dependent pathway (Maurin et al. 2005). Additional signaling pathways might also be involved, for example, enzymatic pathways involved in the NO formation have been implicated in food intake regulation under Thr deprivation (Monda et al. 1998), which may account for the different extents in suppressing food intake by different EAAs. On the other hand, time required for sensing each EAA deficiency could also be different (Gietzen et al. 2007). It may take longer time for mice to sense the deficiency of Lys in the diet.

Because food intake is decreased by EAAs deficiency, we could not ignore the possible contributions from the decreased food intake to the reduced fat mass, in addition to the effect of AA deficiency. The effects of reduced food intake, however, is unlikely to contribute significantly to the decreased fat mass in mice maintained on a diet deficient for any of these five EAAs, based on results obtained in our previous work by including a pair-fed (pf) group to compare the effects of leucine deprivation (Cheng et al. 2010). Consistent with this possibility, Virginia L. Malloy and coworkers showed that Met restriction significantly decreases visceral fat mass, whereas pf group exhibits comparable amounts of visceral fat to control diet-fed rats (Malloy et al. 2006).

Results from our studies (Guo \& Cavener 2007, Cheng et al. 2010, Du et al. 2012) and by others (Zhang et al. 2007, Nairizi et al. 2009, Newgard et al. 2009, Nishimura et al. 2010, Noguchi et al. 2010) suggest a close relationship between AAs and lipid metabolism in mice. Recently, this relationship has also been confirmed in human subjects in Western population by the group of Christopher B. Newgard (Newgard et al. 2009). They used components analysis (PCA) to analyze the difference of metabolites between obese and lean subjects and found that the component showing the strongest differences was composed of a combination of AAs. An association between AAs and obesity in Chinese population, however, has never been tested. In this study, we found that levels of twelve serum amino acids, including four EAAs (Val, Ile, Leu and Phe) and eight non-EAAs (Gly, Cit, bAla, Ala, Glu, Aad, Cys and Tyr), were different between lean and

Published by Bioscientifica Ltd. 
obese groups in Chinese Han population. These results suggest that a close relationship between AAs and BMI is also observed in Chinese Han population, though many different factors affecting AA metabolism and obesity exist between Western and Chinese population. We should also note that this is the first study measuring the most complete AA profile in humans.

In this study, we found levels of four EAAs (Val, Ile, Leu and Phe) are elevated in obese subjects compared with lean controls. Very importantly, elevated levels of the same set of EAAs are also observed in the Western population (Newgard et al. 2009). This is a very exciting finding due to the important roles that EAAs play in metabolic control. These results suggest that these four EAAs might play important roles in the occurrence of human obesity. Consistent with this possibility, deficiency of any of these four EAAs significantly reduces fat mass in mice. We did not see any difference in the levels of the other four EAAs (Thr, Trp, Met or Lys) between obese and lean Chinese. However, it does not necessarily mean that these EAAs do not contribute to obesity, as deficiency of any of these four EAAs also decreases fat mass in mice. Future studies will be required to clarify this issue.

In addition to EAAs, levels of non-EAAs were also compared between lean and obese subjects in Chinese Han population. No changes in levels of His, Met, Orn and Ser were detected between obese and lean groups in Chinese and Western population, indicating that these AAs are unlikely to be associated with obesity. Among eight non-EAAs showing difference between obese and lean humans in Chinese Han population, five of them are also found changed in obese subjects in Western population. These are Gly, Cit, Ala, Glu and Tyr. Therefore, these five non-EAAs might also be involved in human obesity. In addition, we found the levels of bAla, Aad and Cys were also different between these two groups in Chinese population. Besides, levels of Asp, Arg and Pro were not dramatically elevated in Chinese Han population as reported in Western population (Newgard et al. 2009), suggesting a possible unique AA profile between different populations, which might be caused by differences in dietary intake and genetic background.

Taken together, this study showed that the effects of BCAAs deprivation represent a general effect of EAAs deficiency in mice and demonstrated a close relationship between AAs and lipid metabolism in Chinese Han population. These observations provide important hints in understanding nutritional and genetic regulation of obesity and possible intervention strategy by manipulation of dietary amino acids.
Declaration of interest

The authors declare that there is no conflict of interest that could be perceived as prejudicing the impartiality of the research reported.

\section{Funding}

This work was supported by grants from the National Natural Science Foundation (81130076, 81325005, 31271269, 81390350, 81300659 and 81570777), Basic Research Project of Shanghai Science and Technology Commission (13JC1409000); International S\&T Cooperation Program of China (Singapore 2014DFG32470); Research supported by the CAS/ SAFEA international partnership program for creative research teams. F Guo was also supported by the One Hundred Talents Program of the Chinese Academy of Sciences. F Xiao was supported by Youth Innovation Promotion Association CAS and SA-SIBS scholarship program.

\section{Author contribution statement}

F Xiao researched data, wrote, reviewed and edited the manuscript. Y Du, Z Lv and S Chen researched data and contributed to the discussion. $\mathrm{J}$ Zhu and $\mathrm{H}$ Sheng provided research material. F Guo directed the project, contributed to discussion and wrote, reviewed and edited the manuscript.

\section{References}

Attie AD \& Scherer PE 2009 Adipocyte metabolism and obesity. Journal of Lipid Research 50 (Supplement) S395-S399. (doi:10.1194/jlr. R800057-JLR200)

Bubenikova V, Horacek J, Kozeny J, Platilova V, Zavesicka L, Palenicek T \& Hoschl C 2004 The effect of tryptophan depletion on the action of haloperidol in MK-801-treated rats. European Journal of Pharmacology 502 109-116. (doi:10.1016/j.ejphar.2004.08.034)

Cheng Y, Meng Q, Wang C, Li H, Huang Z, Chen S, Xiao F \& Guo F 2010 Leucine deprivation decreases fat mass by stimulation of lipolysis in white adipose tissue and upregulation of uncoupling protein 1 (UCP1) in brown adipose tissue. Diabetes 59 17-25. (doi:10.2337/db09-0929)

Du Y, Meng Q, Zhang Q \& Guo F 2012 Isoleucine or valine deprivation stimulates fat loss via increasing energy expenditure and regulating lipid metabolism in WAT. Amino Acids 43 725-734. (doi:10.1007/ s00726-011-1123-8)

Gietzen DW, Hao S \& Anthony TG 2007 Mechanisms of food intake repression in indispensable amino acid deficiency. Annual Review of Nutrition 27 63-78. (doi:10.1146/annurev.nutr.27.061406.093726)

Guo F \& Cavener DR 2007 The GCN2 eIF2alpha kinase regulates fatty-acid homeostasis in the liver during deprivation of an essential amino acid. Cell Metabolism 5 103-114. (doi:10.1016/j. cmet.2007.01.001)

Hasek BE, Stewart LK, Henagan TM, Boudreau A, Lenard NR, Black C, Shin J, Huypens P, Malloy VL, Plaisance EP, et al. 2010 Dietary methionine restriction enhances metabolic flexibility and increases uncoupled respiration in both fed and fasted states. American Journal of Physiology: Regulatory, Integrative and Comparative Physiology 299 R728-R739. (doi:10.1152/ajpregu.00837.2009)

Hlais S, Reslan DR, Sarieddine HK, Nasreddine L, Taan G, Azar S \& Obeid OA 2012 Effect of lysine, vitamin B(6), and carnitine supplementation on the lipid profile of male patients with hypertriglyceridemia: a 12-week, open-label, randomized, placebo-controlled trial. Clinical Therapeutics 34 1674-1682. (doi:10.1016/j.clinthera.2012.06.019)

Lowell BB \& Bachman ES 2003 Beta-adrenergic receptors, diet-induced thermogenesis, and obesity. Journal of Biological Chemistry $\mathbf{2 7 8}$ 29385-29388. (doi:10.1074/jbc.R300011200) 
Malloy VL, Krajcik RA, Bailey SJ, Hristopoulos G, Plummer JD \& Orentreich N 2006 Methionine restriction decreases visceral fat mass and preserves insulin action in aging male Fischer 344 rats independent of energy restriction. Aging Cell 5 305-314. (doi:10.1111/j.1474-9726.2006.00220.x)

Martin S, Okano S, Kistler C, Fernandez-Rojo MA, Hill MM \& Parton RG 2009 Spatiotemporal regulation of early lipolytic signaling in adipocytes. Journal of Biological Chemistry 284 32097-32107. (doi:10.1074/jbc.M109.002675)

Maurin AC, Jousse C, Averous J, Parry L, Bruhat A, Cherasse Y, Zeng H, Zhang Y, Harding HP, Ron D, et al. 2005 The GCN2 kinase biases feeding behavior to maintain amino acid homeostasis in omnivores. Cell Metabolism 1 273-277. (doi:10.1016/j.cmet.2005.03.004)

Monda M, Viggiano A, Sullo A \& De Luca V 1998 Nitric oxide reduces hypophagia induced by threonine free diet in the rat. Brain Research 808 129-133. (doi:10.1016/S0006-8993(98)00733-1)

Nair KS \& Short KR 2005 Hormonal and signaling role of branchedchain amino acids. Journal of Nutrition 135 1547S-1552S.

Nairizi A, She P, Vary TC \& Lynch CJ 2009 Leucine supplementation of drinking water does not alter susceptibility to diet-induced obesity in mice. Journal of Nutrition 139 715-719. (doi:10.3945/jn.108.100081)

Newgard CB, An J, Bain JR, Muehlbauer MJ, Stevens RD, Lien LF, Haqq AM, Shah SH, Arlotto M, Slentz CA, et al. 2009 A branchedchain amino acid-related metabolic signature that differentiates obese and lean humans and contributes to insulin resistance. Cell Metabolism 9 311-326. (doi:10.1016/j.cmet.2009.02.002)

Nishimura J, Masaki T, Arakawa M, Seike M \& Yoshimatsu H 2010 Isoleucine prevents the accumulation of tissue triglycerides and upregulates the expression of PPARalpha and uncoupling protein in diet-induced obese mice. Journal of Nutrition 140 496-500. (doi:10.3945/jn.109.108977)

Noguchi Y, Nishikata N, Shikata N, Kimura Y, Aleman JO, Young JD, Koyama N, Kelleher JK, Takahashi M \& Stephanopoulos G 2010 Ketogenic essential amino acids modulate lipid synthetic pathways and prevent hepatic steatosis in mice. PLOS ONE 5 e12057. (doi:10.1371/journal.pone.0012057)

Rathore RM, Liaset B, Hevroy EM, El-Mowafi A \& Espe M 2010 Lysine limitation alters the storage pattern of protein, lipid and glycogen in on-growing Atlantic salmon. Aquaculture Research 41 e751-e759. (doi:10.1111/j.1365-2109.2010.02576.x)

Rogers SR \& Pesti GM 1990 The influence of dietary tryptophan on broiler chick growth and lipid metabolism as mediated by dietary protein levels. Poultry Science 69 746-756. (doi:10.3382/ ps.0690746)

Schoenborn V, Heid IM, Vollmert C, Lingenhel A, Adams TD, Hopkins PN, Illig T, Zimmermann R, Zechner R, Hunt SC, et al. 2006 The ATGL gene is associated with free fatty acids, triglycerides, and type 2 diabetes. Diabetes 55 1270-1275. (doi:10.2337/db05-1498)

Singh YS, Anand \& Misra VK 1979 Kidney lipids of weanling rats fed lysine threonine deficient rice diets. Nutrition Reports International 20 75-77.

Smriga M, Murakami H, Mori M \& Torii K 2000 Effects of L-lysine deficient diet on the hypothalamic interstitial norepinephrine and diet-induced thermogenesis in rats in vivo. Biofactors 12 137-142. (doi:10.1002/biof.5520120121)

Wu Y 2006 Overweight and obesity in China. BMJ 333 362-363. (doi:10.1136/bmj.333.7564.362)

Xiao F, Huang Z, Li H, Yu J, Wang C, Chen S, Meng Q, Cheng Y, Gao X, Li J, et al. 2011 Leucine deprivation increases hepatic insulin sensitivity via GCN2/mTOR/S6K1 and AMPK pathways. Diabetes 60 746-756. (doi:10.2337/db10-1246)

Yeaman SJ 1990 Hormone-sensitive lipase - a multipurpose enzyme in lipid metabolism. Biochimica et Biophysica Acta 1052 128-132. (doi:10.1016/0167-4889(90)90067-N)

Zhang P, McGrath BC, Reinert J, Olsen DS, Lei L, Gill S, Wek SA, Vattem KM, Wek RC, Kimball SR, et al. 2002 The GCN2 eIF2alpha kinase is required for adaptation to amino acid deprivation in mice. Molecular Cell Biology 22 6681-6688. (doi:10.1128/MCB.22.19.66816688.2002)

Zhang J, Hupfeld CJ, Taylor SS, Olefsky JM \& Tsien RY 2005 Insulin disrupts beta-adrenergic signalling to protein kinase A in adipocytes. Nature 437 569-573. (doi:10.1038/nature04140)

Zhang Y, Guo K, LeBlanc RE, Loh D, Schwartz GJ \& Yu YH 2007 Increasing dietary leucine intake reduces diet-induced obesity and improves glucose and cholesterol metabolism in mice via multimechanisms. Diabetes 56 1647-1654. (doi:10.2337/db07-0123)

Received in final form 6 September 2016

Accepted 7 September 2016

Accepted Preprint published online 9 September 2016
(๖) 2016 Society for Endocrinology Printed in Great Britain 